\section{Developmental disability in schoolchildren from four Brazilian regions}

Rev Bras Psiquiatr. 2014;36:273-274

doi:10.1590/1516-4446-2013-1342

Child developmental disability (CDD) is an emerging global health priority. More than 140 million children live with a disability in unfavorable socioeconomic conditions worldwide. ${ }^{1}$

A review including 80 papers documented a lack of high-quality research into CDD. Few of these studies were conducted in Latin America, and the only Brazilian epidemiological study was focused on hearing loss. ${ }^{2} \mathrm{~A}$ review of intellectual disabilities found no prevalence studies conducted in Brazil. ${ }^{3}$ Moreover, until now, no study has addressed CDD in different areas of the country. This is especially problematic in a large country such as Brazil, with great differences between regions.

Another important study on disability was carried out with 191,199 children from 18 low-income countries and demonstrated a high prevalence of disability (median: 23\%; range: $3-48 \%$ ), according to the Ten Question Screen (TQ). Of the 18 countries included, only three were in Latin America. ${ }^{1}$ The absence of epidemiological data on CDD in Brazil is a barrier to the development of effective policies.

In the last few years, the Brazilian government has launched several programs related to CDD, but the magnitude of the problem is still unknown. Here we present data from the first study to address CDD in different geographic regions of Brazil. This crosssectional study included a random sample of 905 children (6-9 years old) recruited from public elementary schools in four Brazilian municipalities in four different regions
(North, Northeast, Center-West, and Southeast), whose parents were interviewed face-to-face using the TQ. The TQ consists of 10 yes/no questions that screen for functional limitations in the domains of speech, cognition, hearing, vision, motor/physical impairment, and seizure disorders. This instrument is based on parent report and has been validated in several countries using a cutoff point of one or more positive items. It is applicable to children aged 2-9 years in most cultural settings, and is one of the most commonly used instruments worldwide. ${ }^{2,4}$ The local Research Ethics Committee approved the project.

Using a cutoff point of one or more positive TQ items, the prevalence of child developmental disability was $38.5 \%$ (Table 1). There were no differences in rates among the studied regions $(p=1.9)$.

The prevalence of CDD in our sample was higher than the median obtained in the most comprehensive international study. This rate is higher than that estimated in Jamaica (24\%), similar to that found in Suriname $(39 \%)$, and slightly lower than that found in Belize $(44 \%){ }^{1}$ It is noteworthy that the TQ is a screening tool, and no standardized procedure was carried out to confirm true disability. Nevertheless, this estimate reveals a high proportion of children who probably need special care and experience consequences associated with disabilities, such as stigmatization and lack of opportunities.

In conclusion, identification of CDD is important to help minimize obstacles to healthy child development. This is a first step to help focus government attention on the need to develop appropriate social, health, and educational programs. Given the recent growth in economic development in Brazil, the country now has more resources, and these data should guide the development of policies and the fair allocation of funds.

\section{Rosane Lowenthal, ${ }^{1}$ Livia Zaqueu, ${ }^{2}$ Luis A. Rohde, ${ }^{3}$} Jair Mari, ${ }^{4}$ Cristiane S. Paula ${ }^{2,4}$

${ }^{1}$ Mental Healthcare Center (CAISM), Santa Casa de São Paulo, São Paulo, SP, Brazil. ${ }^{2}$ Developmental Disorders Program, Universidade Presbiteriana Mackenzie, São Paulo, SP, Brazil. ${ }^{3}$ Department of Psychiatry, Universidade Federal do Rio Grande do Sul (UFRGS), Porto Alegre, RS, Brazil. ${ }^{4}$ Department of Psychiatry, Universidade Federal de São Paulo (UNIFESP), São Paulo, $S P$, Brazil

Submitted Dec 16 2013, accepted Jan 202014.

Table 1 Frequency of disabilities in children aged 6 to 9 years from four Brazilian regions $(n=905)$

\begin{tabular}{|c|c|c|}
\hline TQ question & $\mathrm{n}$ & $\%$ positive \\
\hline 1. Developmental milestones & 42 & 4.6 \\
\hline 2. Vision & 122 & 13.5 \\
\hline 3. Hearing & 50 & 5.6 \\
\hline 4. Comprehension & 42 & 4.7 \\
\hline 5. Movement & 27 & 3.0 \\
\hline 6. Seizure & 25 & 2.8 \\
\hline 7. Learning & 47 & 5.2 \\
\hline 8. Speech & 23 & 2.5 \\
\hline 9. Speech and communication & 61 & 6.7 \\
\hline 10. Intellectual impairment & 145 & 16.0 \\
\hline $\mathrm{TQ}+$ & 348 & 38.5 \\
\hline
\end{tabular}

$\mathrm{TQ}=$ Ten Question Screen. 


\section{Disclosure}

LAR has been a member of the speakers' bureau/ advisory board and/or acted as a consultant for Eli-Lilly, Janssen-Cilag, Novartis, and Shire in the last 3 years; he receives authorship royalties from Oxford Press and ArtMed; he has also received travel awards from Shire for his participation in the 2014 APA meeting; the ADHD and Juvenile Bipolar Disorder Outpatient Programs chaired by him have received unrestricted educational and research support from the following pharmaceutical companies in the last three years: Eli-Lilly, JanssenCilag, Novartis, and Shire. The other authors report no conflicts of interest.

\section{References}

1 Gottlieb CA, Maenner MJ, Cappa C, Durkin MS. Child disability screening nutrition and early learning in 18 countries with low and middle incomes: data from the third round of UNICEF's Multiple Indicator Cluster Survey (2005-06). Lancet. 2009;374:1831-9.

2 Maulik PK, Darmstadt GL. Childhood disability in low and middle incomes countries: overview of screening, prevention, services, legislation, and epidemiology. Pediatrics. 2007;120:S1-55.

3 Mercadante MT, Evans-Lacko S, Paula CS. Perspectives of intellectual disability in Latin American countries: epidemiology, policy, and services for children and adults. Curr Opin Psychiatry. 2009;22:469-74.

4 Durkin MS, Wang W, Shrout P, Zaman SS, Hasan ZM, Desai P, et al. Evaluating a ten questions screen for childhood disability: reliability and internal structure in different cultures. J Clin Epidemiol. 1995;48:657-66. 